Nota científica

(Short communication)

\title{
PRIMER REPORTE DEL ESCARABAJO ACANTHODERES FUNERARIA (BATES) (COLEOPTERA: CERAMBYCIDAE) EN EL AGAVE SILVESTRE AGAVE CUPREATA (TREL. \& BERGER) EN GUERRERO, MÉXICO
}

\author{
FIRST REPORT OF THE ACANTHODERES FUNERIA (BATES) (COLEOPTERA: \\ CERAMBYCIDAE) BEETLE IN THE WILD AGAVE CUPREATA (TREL. \& BERGER) AT \\ GUERRERO, MEXICO
}

\author{
EDUARDO SÁNCHEZ JIMÉNEZ ${ }^{1}$, DIANA IVETH ORBE DÍAZ², YANET ROMERO², JEIRY TORIBIO \\ JIMÉNEZ ${ }^{2 *}$
}

\begin{abstract}
${ }^{1}$ Doctorante, Centro de Estudios e Investigación en Desarrollo Sustentable, Universidad Autónoma del Estado de México. Mariano Matamoros, Col. Universidad. 1007., C.P. 50130, Estado de México, México. <mayor_sanchez@hotmail.com> ${ }^{2}$ Facultad de Ciencias Químico Biológicas, Universidad Autónoma de Guerrero, Ciudad universitaria, Chilpancingo, Gro., México.<ivethod11@gmail.com>; <yanetromero7@gmail.com>; <jeiryjimenez2014@gmail.com>

*Autor de correspondencia: <jeiryjimenez2014@gmail.com>
\end{abstract}

Recibido: 08/09/2018; aceptado: 27/08/2019; publicado en línea: 20/09/2019

Editor responsable: Jesús Romero Nápoles

Sánchez-Jiménez, E., Orbe-Díaz, D. I., Romero, Y., Toribio-Jiménez, J. (2019) Primer reporte del escarabajo Acanthoderes funeraria (Bates) (Coleoptera: Cerambycidae) en el agave silvestre Agave cupreata (Trel. \& Berger) en Guerrero, México. Acta Zoológica Mexicana (nueva serie), 35, 1-4. https://doi.org/10.21829/azm.2019.3502072

RESUMEN. Acanthoderes funeraria, es un escarabajo que se alimenta de las hojas de plantas de agave y causa daños graves e irreversibles en las plantas. Se hizo una colecta el 20 de junio de 2018, en el poblado de Petaquillas, Guerrero, para esto se revisaron plantas jóvenes y adultas enfermas, se logró colectar manualmente 60 especímenes y se tomaron fotos, las cuales se enviaron a la CONABIO para su identificación, la cual confirmó la especie como A. funeraria y registró la presencia de ésta para el estado de Guerrero; otros especímenes se encuentran depositados en la colección entomológica de la Facultad de Ciencias Químico Biológicas de la Universidad Autónoma de Guerrero con número de registro 020219fcqb. Este escarabajo se reporta por vez primera sobre agaves silvestres de A. cupreata en el estado de Guerrero, México; además, se describen los daños que éste causa a las plantas.

Sánchez-Jiménez, E., Orbe-Díaz, D. I., Romero, Y., Toribio-Jiménez, J. (2019) First report of the Acanthoderes funeria (Bates) (Coleoptera: Cerambycidae) beetle in the wild Agave cupreata (Trel. $\&$ Berger) at Guerrero, Mexico. Acta Zoológica Mexicana (nueva serie), 35, 1-4. https://doi.org/10.21829/azm.2019.3502072

ABSTRACT. Acanthoderes funeraria, is a beetle that feeds on the leaves of agave plants and causes serious and irreversible damage to plants. A collection of insects was made on June 20, 2018, in the town of Petaquillas, Guerrero. Young and adult sick plants were checked, then 60 specimens were collected by hand and photos were taken and images were sent to CONABIO for identification, which confirmed the species as A. funeraria and it recorded the presence in the state of Guerrero; other specimens were deposited in the 
Entomological Collection of the Faculty of Biological Sciences of the Autonomous University of Guerrero with registration number $020219 \mathrm{fcqb}$. This beetle for the first time is recorded on wild agaves of A. cupreata in the state of Guerrero, Mexico; moreover, the damage to the plants is described.

El género Agave L. incluye plantas adaptadas a condiciones de aridez (Domínguez et al., 2008) y se estima alrededor de 200 especies, de éstas 159 se encuentran en México (García-Mendoza, 2002; Velasco et al., 2009). Entre las especies de mayor importancia económica, por su uso para la elaboración de bebidas fermentadas o destiladas en México, destacan Agave salmiana Otto ex Salm-Dyck (maguey de pulque), $A$. angustifolia Haw. (espadín), A. tequilana F.A.C. Weber (tequila), A. lechuguilla Torr. (lechuguilla) y $A$. cupreata Trel. \& Berger (papalote) (García, 2011). Agave cupreata es una especie endémica que se desarrolla en la Cuenca del Balsas-Mezcala en el estado de Guerrero y se ha reportado también en Guanajuato, Jalisco, Michoacán, Morelos, Oaxaca y Querétaro (IIlsley, 2008; García, 2010); es la especie de mayor interés social y económico en Guerrero por la demanda en la elaboración de mezcal (Alonso, 2007). Para la producción del mezcal en Guerrero, en las zonas proveedoras de agave, el kilo se cotiza en $\$ 15.00$ aproximadamente y se requieren $10 \mathrm{~kg}$ de agave para producir un $1 \mathrm{~L}$ de mezcal. En promedio una fábrica de mezcal artesanal produce anualmente 3,500 L de mezcal durante los meses de febrero a junio, con un procesamiento de cerca de $35,000 \mathrm{~kg}$ de agave y un costo de producción general de $\$ 525,000.00$ por ciclo productivo. Entre los factores que limitan la preservación del agave se encuentran el crecimiento muy lento, tasas bajas de polinización, baja viabilidad de las semillas e insectos plaga, como el picudo del agave Scyphophorus acupunctatus (Solís-Aguilar et al., 2001) o el escarabajo funerario del maguey A. funeraria y enfermedades como la bacteria Erwinia carotovora (García et al., 2010).

Acanthoderes funeraria Bates es un cerambícido registrado en las plantaciones de agave que causa problemas en los estados de Guanajuato, Jalisco, Michoacán y Querétaro (IIlsley, 2008). En Hidalgo, se reportó por primera vez en A. salmiana (Olivares-Orozco et al., 2017). Adultos de esta especie se encontraron en poblaciones silvestres de $A$. cupreata en el poblado de Petaquillas, municipio de Chilpancingo de los Bravo (17 28' 17.2" N, 99 23' 53.6" W). Se observó como este escarabajo causa daños irreversibles en las pencas (la piña del agave) y causó la muerte a las plantas, por lo que se ha denominado a ese lugar como "el cementerio del A. cupreata" (Fig. 1A-D). Se colectaron manualmente 60 especímenes adultos entre las pencas el 20 de junio de 2018 y se trasladaron al laboratorio en un frasco seco y estéril para su estudio y para tomar fotografías. Las imágenes obtenidas se enviaron a la Comisión Nacional de Biodiversidad (CONABIO) para su identificación, misma que confirmó a la especie como A. funeraria. Los especímenes muertos se preservaron en alcohol (70\%) y refrigeración en el Laboratorio de Microbiología Molecular y Biotecnología Ambiental y se enviaron 20 especímenes al laboratorio de Entomología a cargo del Dr. Antonio Juan Cortés Guzmán para formar parte de su colección, con número de colecta 020219fcqb, ambos laboratorios pertenecen a la Facultad de Ciencias Químico Biológicas de la Universidad Autónoma de Guerrero. Con este primer reporte se pretende definir una línea de investigación con la finalidad de implementar estrategias de control biológico para proteger las poblaciones de silvestres de A. cupreata en Guerrero.

Se observó un total de 60 escarabajos y en promedio se encontraron dos especímenes por planta en la misma visita, tanto en plantas adultas como jóvenes; en promedio los escarabajos median $2 \mathrm{~cm}$ de largo x $0.7 \mathrm{~cm}$ de ancho (Fig. 2A-C). Olivares-Orozco et al. (2017) reportaron seis especímenes por planta y describieron lesiones encontradas en A. salmiana que coinciden con las que se observaron sobre A. cupreata. Estas lesiones causadas por los adultos en la superficie de las hojas dejaron una señal característica, que en la mayoría de los casos fueron perpendiculares al eje de la penca, con cambio de color de verde a café hasta causar la muerte de las hojas; en tanto que las larvas barrenan la piña provocando la muerte total de la planta (Fig. 1C, D). Los estragos que este escarabajo ha causado en las plantas silvestres de A. cupreata, en el ejido de Petaquillas y en áreas aledañas, atentan al desarrollo sustentable, disponibilidad y acceso del agave, 
principal insumo del sistema productivo agave-mezcal, por lo que debe estudiarse y definir estrategias de manejo para lograr una mejor conservación de la especie de $A$. cupreata.
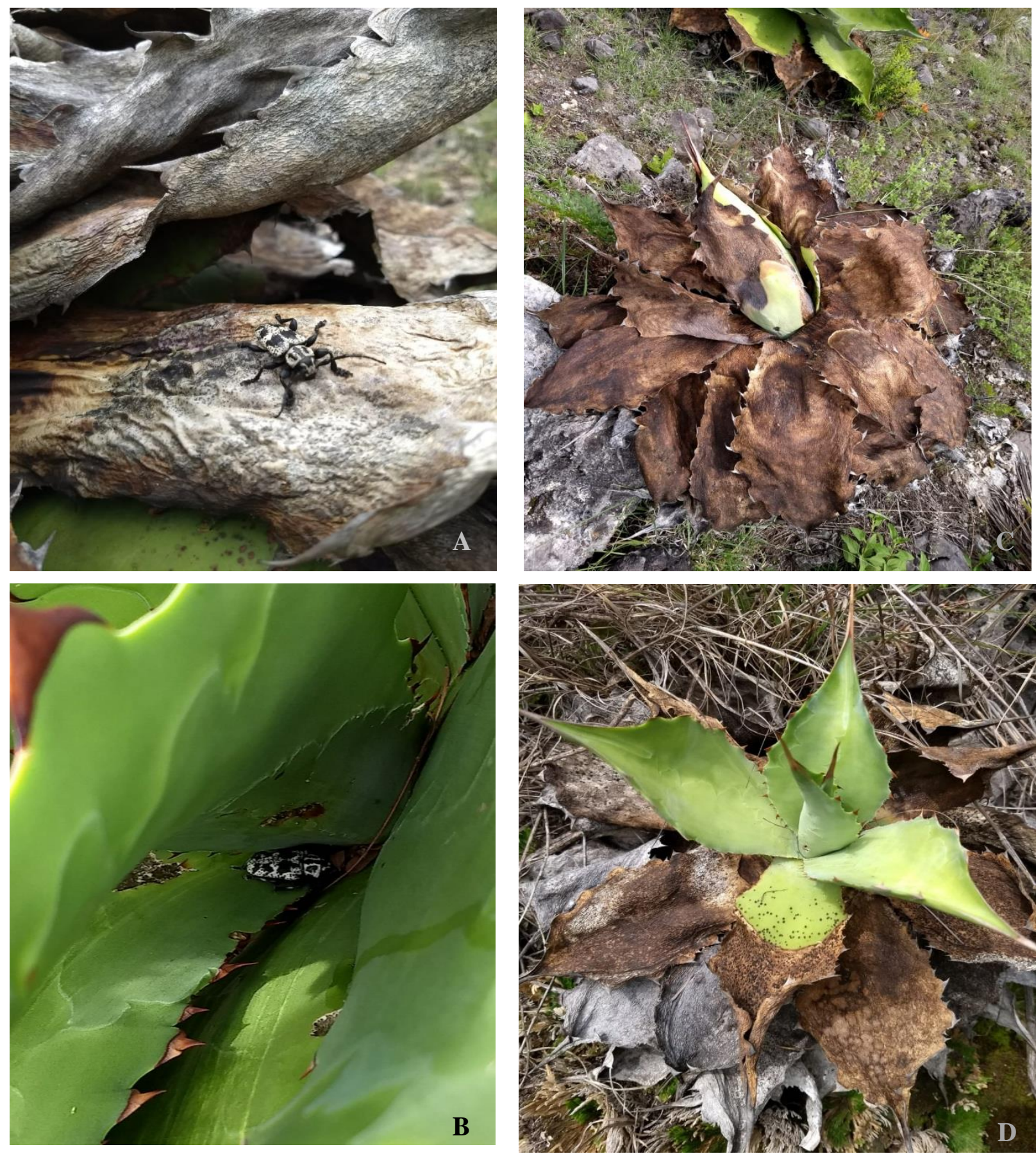

Figura 1. Adulto de A. funeraria en plantas de A. cupreata silvestres (A y B), daños ocasionados a las plantas jóvenes (C y D). 


\section{LITERATURA CITADA}

Alonso, C. (2007) Manejo campesino en el sistema de producción del maguey Papalote (Agave cupreata Trel. \& A. Berger.), para la elaboración de mezcal en la región de Chilapa, Gro. Tesis de Maestría. Puebla, México. Colegio de Postgradudados, Campus Puebla.

Domínguez, M., Alpuche A., Vasco N., Pérez, E. (2008) Efecto de citocininas en la propagación in vitro de agaves mexicanos. Revista Fitotecnia Mexicana, 31 (4), 317-322.

García-Mendoza, A. (2002) Distribution of Agave (Agavaceae) in México. Cactus and Succulent Journal, 74, 177-187.

García, A. (2011) Flora del Valle de Tehuacán-Cuicatlán. (Fascículo 88:1-95). CC. de Universidad Nacional Autónoma de México. Instituto de Biología. SNIB-CONABIO.

García, J., Méndez, S., Talavera, D. (2010) El género Agave spp. en México: Principales usos de importancia socioeconómica y agroecológica. Revista Salud Pública y Nutrición, 5, 109-121.

IIlsley, C. (2008) Manejo campesino sustentable del maguey papalote del Chilapan. Fase II. Grupo de Estudios Ambientales AC. Informe final SNIB-CONABIO proyecto No. ES004. México D.F. Disponible en: www.inaturalist.org

Olivares-Orozco, Javier, Ramírez-Sánchez, S. E., Jiménez-Aguilar, A., Guerrero-Andrade, O., Montiel-Salero, D., Rodríguez-Diego, J. G. (2017) Notificación de Acanthoderes funeraria (Bates) sobre dos tipos de Agave salmiana (Otto ex Salm-Dyck) en Hidalgo, México. Revista de Protección Vegetal, 32 (2) (Online).

Solís-Aguilar, J. F., González-Hernández, H., Leyva-Vázquez, J. L., Equihua-Martínez, A., FloresMendoza, F. J., Martínez-Garza, Á. (2001) Scyphophorus acupunctatus gyllenhal, plaga del agave tequilero en Jalisco, México. Agrociencia, 35, 663-670.

Velasco, E., Zamora, H., Espinosa, C., Sampayo, F., Moreno, S. (2009) Modelos Predictivos para la Producción de Productos Forestales No Maderables: Agaves Mezcaleros. Manual Técnico Núm. 3. CENID-COMEF. INIFAP, México, D. F. México, 60 p. 